\title{
Component-Resolved IgE Profiles in Austrian Patients with a Convincing History of Peanut Allergy
}

\author{
Daniela Ackerbauer ${ }^{\mathrm{a}}$ Merima Bublin ${ }^{\mathrm{a}}$ Christian Radauer ${ }^{\mathrm{a}}$ Eva-Maria Varga ${ }^{\mathrm{e}}$ \\ Christine Hafner $^{f}$ Christof Ebner $^{d}$ Zsolt Szépfalusi ${ }^{c}$ Renate Fröschl ${ }^{b}$ \\ Karin Hoffmann-Sommergruber ${ }^{a}$ Thomas Eiwegger ${ }^{c}$ Heimo Breiteneder ${ }^{a}$ \\ Departments of a Pathophysiology and Allergy Research, ${ }^{\mathrm{b}}$ Medical and Chemical Laboratory Diagnostics and \\ 'Pediatrics and Adolescent Medicine, Medical University of Vienna, dAllergy Clinic Reumannplatz, Vienna, \\ e Department of Paediatrics, Respiratory and Allergic Disease Division, Medical University, Graz, and ${ }^{\mathrm{f} K a r l}$ \\ Landsteiner Institute for Dermatological Research, St. Poelten, Austria
}

\section{Key Words}

Allergy · Allergens · Ara h $2 \cdot$ Ara h $8 \cdot$ Component-resolved diagnosis · Food allergy $\cdot$ Peanut allergy

\begin{abstract}
Background: Peanut allergy develops after primary sensitization to peanut allergens and/or lgE cross-sensitization with homologous allergens from various plants. Therefore, heterogeneous patterns of sensitization to individual peanut allergens are observed in different countries. The aim of this study was to examine the IgE sensitization patterns of Austrian peanut-allergic patients. Methods: Sera from 65 peanut-allergic patients and 20 peanut-tolerant atopics were obtained in four Austrian allergy clinics. Sensitization patterns against peanut allergens Ara $h$ 1-3, 6, 8 and 9 were identified by ImmunoCAP and ImmunoCAP ISAC. Results: Austrian peanut-allergic patients were sensitized to Ara $\mathrm{h} 2$ and $6(71 \%)$, followed by Ara h 1 (62\%), Ara h 8 (45\%), Ara h 3 (35\%) and Ara h 9 (11\%). All sera containing Ara h 2-specific IgE were also positive for Ara h 6, with Ara h 6-specific lgE levels significantly $(p<0.05)$ higher compared with Ara h 2. Twelve percent displayed IgE reactivity exclusively to Ara h 8. Peanut extract and Ara h 8 showed low diagnostic spec-
\end{abstract}

\begin{tabular}{ll}
\hline KARGER 125: & $\begin{array}{l}\text { (2) 2015 S. Karger AG, Basel } \\
1018-2438 / 15 / 1661-0013 \$ 39.50 / 0\end{array}$ \\
$\begin{array}{l}\text { E-Mail karger@karger.com } \\
\text { www.karger.com/iaa }\end{array}$ & $\begin{array}{l}\text { This is an Open Access article licensed under the terms of the } \\
\text { Creative Commons Attribution-NonCommercial 3.0 Un- } \\
\text { ported license (CC BY-NC) (www.karger.com/OA-license), } \\
\text { applicable to the online version of the article only. Distribu- } \\
\text { tion permitted for non-commercial purposes only. }\end{array}$
\end{tabular}

ificities of 25 and 10\%, respectively. The other peanut allergens showed $100 \%$ specificity. Diagnostic sensitivities determined by ImmunoCAP ISAC and ImmunoCAP were highly similar for Ara h 2, 3 and 8. Conclusions: The majority of symptomatic peanut-allergic patients are sensitized to Ara $h$ 2 and Ara h 6 . In peanut-symptomatic patients with additional birch pollen allergy, other peanut allergens, especially Ara $h 8$, should be tested when IgE reactivity to Ara $h 2$ is absent.

ㄷ) 2015 S. Karger AG, Basel

\section{Introduction}

By using component-resolved diagnosis (CRD) heterogeneous patterns of sensitization to peanut allergens have been reported across different areas of the world. Furthermore, marked differences in the allergen recognition profiles could be related to distinct clinical manifestations of peanut allergy. The peanut seed storage proteins Ara h 1, Ara h 2, Ara h 3 and Ara h 6 are largely recognized

D.A. and M.B. contributed equally to this work.
Correspondence to: Dr. Merima Bublin

Department of Pathophysiology and Allergy Research Medical University of Vienna

Waehringer Guertel 18-20, AT-1090 Vienna (Austria)

E Mail merima.bublin@meduniwien.ac.at 
as the major allergens in patients who are primarily sensitized to peanut. Among them, Ara h 2 has been described as the most important peanut allergen, with a sensitization rate of $42-100 \%$ depending on the study population, whereas the sensitization rate to Ara h 1 and Ara h 3 is lower, in the range of $30-80$ and $16-61 \%$, respectively [16]. The diagnostic value of Ara $\mathrm{h}$ 6, which has only recently become available on ImmunoCAP ISAC, has been shown to be equivalent to Ara $\mathrm{h} 2$ with a positive predictive value of 95\% [7]. Although only Ara h 2 is considered as a predictor of clinical reactivity to peanut [8-10], polysensitization to Ara h 2 and Ara h 1 and/or Ara h 3 appeared to be predictive of more severe reactions [11-13].

Ara h 8, a homologue of the major birch pollen allergen Bet v 1, and Ara h 5, a profilin, are mostly involved in pollen-associated peanut allergy due to cross-reactivity with their homologues in birch and grass pollen. Consequently, high sensitization rates to Ara h 8 were primarily found in patients allergic to Fagales tree pollen such as birch in Central and Northern Europe (65-70\%) [2, 3, 5] or alder in Japan (42\%) [6]. Ara h 8 sensitization usually indicates tolerance to peanuts or occasionally only causes mild oral symptoms [14]. Sensitization rates to profilin among peanut-sensitized patients were reported to be $3.3 \%$ in the USA, $9-16 \%$ in Northern and Central Europe, and $24 \%$ in Spain $[5,11]$.

The nonspecific lipid-transfer protein Ara h 9 is involved in the so-called nsLTP-syndrome, mainly in peach-allergic patients from the Mediterranean area, but also in some nsLTP-sensitized patients from Central Europe [5]. A recent study showed that asymptomatic peanut-sensitized children from sub-Saharan Africa had IgE almost exclusively specific to cross-reactive carbohydrate determinants (CCDs) induced by glycoproteins from parasitic Schistosoma species [15].

Although the oral food challenge is considered to be the most accurate test for the diagnosis of peanut allergy, evaluating IgE binding to individual peanut allergens provides additional diagnostic information over peanut extract testing, such as the differentiation of IgE binding to allergens predictive of clinically relevant peanut sensitization or to cross-reactive peanut allergens with a smaller risk of severe reactions [16].

To date, no information regarding $\mathrm{CRD}$ and clinical characteristics of peanut-allergic patients in the Austrian population has been available. Therefore, we investigated the profile of IgE antibody responses from peanut-allergic patients to individual peanut allergens and compared them with the IgE response to total peanut allergen extract. Furthermore, CRD results obtained by traditional singleplexed ImmunoCAP were compared with those obtained by the multiplexed ImmunoCAP ISAC microarray technology.

\section{Methods}

\section{Study Population}

Serum samples from 65 patients (33 adolescents/adults and 32 children) with a convincing clinical history of peanut allergy and 20 peanut-tolerant atopic patients were evaluated in this study. Subjects were enrolled in four Austrian specialized outpatient allergy clinics. Peanut allergy was assessed based on the combination of a clinically convincing history and sensitization to peanut (positive skin prick test or positive sIgE to peanut). Symptoms suggestive of allergy were classified into groups according to Sampson et al. [17]. Two of the patients had no detectable peanut-specific IgE but a positive clinical history of peanut allergy. An additional control group consisted of 8 nonatopic subjects. At the time of inclusion, all control patients indicated consuming peanut regularly without allergic symptoms. Ethical approval was obtained by the respective institutional review boards and signed informed consent was obtained from the patients. The clinical data and demographic and clinical characteristics of the two groups are summarized in table 1.

\section{Serological Analysis}

All sera were subjected to allergen-specific IgE determination by using the ImmunoCAP and ImmunoCAP ISAC 112 according to the manufacturer's recommendations. Total IgE, peanut extract-specific IgE and IgE reactivity to individual peanut individual allergens rAra h 1, rAra h 2, rAra h 3, rAra h 8 and rAra h 9 were tested using the ImmunoCAP system (Thermo Fisher Scientific, ImmunoDiagnostics, Uppsala, Sweden). The measuring range was between 0 and $100 \mathrm{kU}_{\mathrm{A}} / \mathrm{l}$. IgE antibody concentration $>100 \mathrm{kU}_{\mathrm{A}} / \mathrm{l}$ were assigned the value of $100 \mathrm{kU}_{\mathrm{A}} / 1$ for statistical evaluations. At the time of the study, the ImmunoCAP ISAC microarray contained 112 allergens, including the following peanut allergens: rAra h 1, rAra h 2, rAra h 3, nAra h 6, rAra h 8 and rAra h 9. MUXF was used as a marker of sensitization to CCDs and Phl p 12, and Bet $\mathrm{v} 2$ as markers of profilin sensitization. IgE levels $>0.35 \mathrm{kU}_{\mathrm{A}} / \mathrm{l}$ for the ImmunoCAP and $>0.3$ ISU-E for the ImmunoCAP ISAC were considered positive.

\section{Statistics}

The nonparametric Wilcoxon rank-sum test was applied to compare the distributions of unpaired scale variables. Accordingly, the Wilcoxon signed-rank test was used in paired situations. Correlations were calculated by the Spearman's rank correlation coefficient. Absolute coefficients $>0.5$ were considered to be moderate and $>0.7$ as strong correlation. $\mathrm{p}$ values $<0.05$ were considered significant. Fisher's exact test was employed to compare groups with binary outcome (anaphylaxis/no anaphylaxis) and low frequencies. Sensitivities were calculated as TP/(TP + FN), where TP is the number of true positives (patients with peanut allergy and positive CAP/ISAC results) and $\mathrm{FN}$ is the number of false negatives (patients with peanut allergy and negative CAP/ISAC results). Specificities were calculated as $\mathrm{TN} /(\mathrm{TN}+\mathrm{FP})$, where a true negative $(\mathrm{TN})$ result is defined as a patient without a reported peanut allergy and negative CAP/ISAC results, and a false positive
Ackerbauer et al. 
Table 1. Clinical data of Austrian peanut-allergic and peanut-tolerant patients

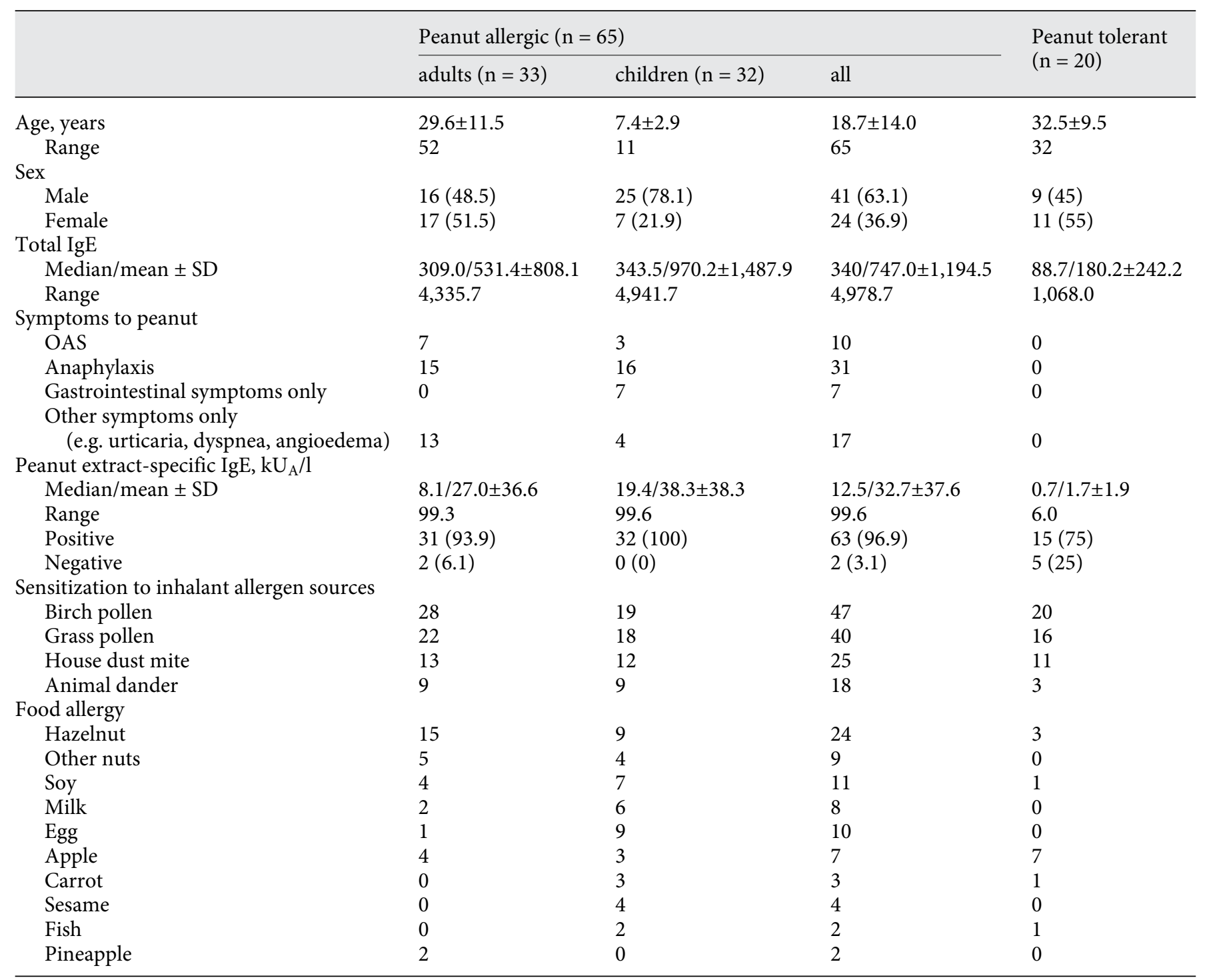

Values are presented as $\mathrm{n}(\%)$ or mean $\pm \mathrm{SD}$, unless otherwise indicated.

(FP) as a patient without peanut allergy but with positive CAP/ ISAC. The range is a measure of dispersion and is calculated by subtracting the minimum from the maximum value. Data analysis was performed using SPSS (SPSS Inc., Chicago, Ill., USA).

\section{Results}

\section{Patient Characteristics}

The study population, comprising 65 Austrian peanut-allergic patients, included 33 adults (mean age
$29.6 \pm 11.5$ years) and 32 children (mean age $7.4 \pm 2.9$ years). There was a female predominance in adults and a male predominance in children. Table 1 summarizes the patients' demographical and clinical characteristics. All 65 patients were sensitized to one or more aeroallergen source, such as birch and grass pollen, house dust mite or animal dander. In addition to peanut allergy, patients had allergy to hazelnut (37\%), soy (17\%), other nuts, such as almond, walnut and/or cashew (14\%), apple (11\%) and sesame seeds (6\%). Ten patients reported symptoms after exposure to peanut localized to the oral 
cavity (itching lips, throat and face), 24 patients had gastrointestinal symptoms (abdominal pain, diarrhea, vomiting) or other systemic reactions (e.g. urticaria, angioedema, dyspnea). Of 65 patients with clinical signs of peanut allergy, 31 fulfilled the criteria of anaphylaxis (i.e. skin symptoms and involvement of the respiratory and/or gastrointestinal tract) following the consumption of traces of peanut. Of those, 11 had asthma, 20 were also sensitized to other food and 28 to inhalant allergen sources. No anaphylactic reactions to foods other than peanut were reported in the history. However, most of the patients with peanut-induced anaphylaxis were also sensitized to birch and/or grass pollen with clinical symptoms of pollen allergy. In addition, 20 peanut-tolerant atopic controls with a mean age of $32.5 \pm 9.5$ years were included. Three patients had allergy exclusively to birch pollen, 5 patients had both birch and grass pollen allergy and 12 patients had additional house dust mite and/or animal dander allergy in addition to birch and grass pollen allergy. Nine patients experienced Bet $\mathrm{v}$ 1 -related oral allergy syndrome (OAS) to apple (35\%), hazelnut (15\%), soy or carrot (5\% each).

Performance of Two Commercially Available in vitro Techniques, ImmunoCAP and ImmunoCAP ISAC, in the Diagnosis of Peanut Allergy

Conventional ImmunoCAP analysis detected peanut extract specific-IgE in 63 (97\%) peanut-allergic patients and in $15(75 \%)$ atopic controls, resulting in high sensitivity but low specificity of the commercial ImmunoCAP peanut extract. Nevertheless, the control group showed significantly lower levels $(p<0.001)$ of peanut-specific IgE at a mean level of $1.7 \mathrm{kU}_{\mathrm{A}} / \mathrm{l}$ (range 6.0) compared with the peanut-allergic group with a mean level of $32.7 \mathrm{kU} \mathrm{A}_{\mathrm{A}} / \mathrm{l}$ (range 99.6; table 3). Peanut-allergic children had higher peanut-specific IgE levels than peanut-allergic adults (mean $38.3 \mathrm{kU}_{\mathrm{A}} / \mathrm{l}$, range 99.6 vs. mean $27.0 \mathrm{kU}_{\mathrm{A}} / 1$, range 99.3).

Peanut-specific IgE, as quantified by traditional ImmunoCAP, was moderately but significantly correlated $(\mathrm{p}<0.01)$ with IgE specific to Ara h 1 (CAP: $\mathrm{r}=0.63$ and ISAC: $\mathrm{r}=0.55)$, Ara h 2 (CAP: $\mathrm{r}=0.66$ and ISAC: $\mathrm{r}=$ 0.63 ), Ara $h 3$ (CAP: $r=0.67$ and ISAC: $r=0.65$ ) and Ara h 6 (ISAC: $r=0.64$ ). No correlation between peanut-specific IgE and IgE specific to Ara h 8 or Ara h 9 was observed.

A high degree of correlation was demonstrated when comparing the ISAC and CAP data among each other for all 5 peanut allergen components, with particularly high correlation coefficients in the cases for Ara h $2(r=0.95)$,
Table 2. CRD analysis comparing ImmunoCAP and ImmunoCAP ISAC: the number of positive subjects and levels of allergen-specific IgE antibodies in peanut-allergic subjects

\begin{tabular}{lllll}
\hline Allergen & $\begin{array}{l}\text { CAP-specific IgE data } \\
\left(>0.35 \mathrm{kU}_{\mathrm{A}} / \mathrm{l}\right)\end{array}$ & $\begin{array}{l}\text { ISAC-specific IgE data } \\
(>0.3 \text { ISU-E })\end{array}$ \\
\hline Peanut & & & - \\
$\quad$ extract & $63(97)$ & $12.5 / 32.7(99.6)$ & - & \multicolumn{1}{l}{} \\
Ara h 1 & $44(68)$ & $3.8 / 20.28(99.8)$ & $40(62)$ & $6.6 / 19.4(167.4)$ \\
Ara h 2 & $44(68)$ & $9.0 / 30.9(99.4)$ & $46(71)$ & $7.6 / 17.4(107.7)$ \\
Ara h 3 & $25(39)$ & $2.6 / 14.2(99.6)$ & $23(35)$ & $4.5 / 22.4(165.1)$ \\
Ara h 8 & $28(43)$ & $2.3 / 12.0(99.5)$ & $29(45)$ & $3.0 / 11.2(79.9)$ \\
Ara h 9 & $10(15)$ & $1.4 / 5.2(31.0)$ & $7(11)$ & $1.5 / 2.5(6.4)$ \\
Ara h 6 & - & - & $46(71)$ & $17.6 / 36.0(177.4)$
\end{tabular}

Values are presented as $\mathrm{n}$ of positive (\%) and median/mean (range).

followed by Ara h $1(\mathrm{r}=0.93)$, Ara h $8(\mathrm{r}=0.91)$ and Ara h $3(r=0.86$; fig. 1 ; table 2$)$. A weaker, although still significant correlation between the two methods was detected for Ara h $9(\mathrm{r}=0.65)$. All correlations were significant at the 0.01 level. None of the sera from nonatopic controls reacted with either the extract or with individual peanut allergens tested by ImmunoCAP and ImmunoCAP ISAC.

Specific IgE was detected against at least one of the five tested peanut allergens by ImmunoCAP (Ara $h 1$, Ara $h$ 2, Ara h 3, Ara h 8 and Ara h 9) in 62 of 65 (95\%) sera of patients with peanut allergy. Sera of 59 (91\%) peanut-allergic subjects contained specific IgE against at least one of the six peanut allergens by ImmunoCAP ISAC and of $62(95 \%)$ when sera containing Phl p 12 or $\mathrm{MUXF}_{3}$-specific IgE as markers of sensitization to profilin or CCDs were included. The high diagnostic sensitivity was however not accompanied by diagnostic specificity, as all sera from peanut-tolerant patients recognized pollen-associated allergens such as Ara h 8 (95\%) and/or Phl p 12 and CCDs.

All peanut-tolerant but birch pollen-allergic subjects had IgE specific for Ara $\mathrm{h} 8$ with a mean of 9.3 versus 11.2 ISU-E for peanut-allergic subjects, resulting in very low specificity for this allergen. All sera containing Ara $h$ 8-specific IgE also had Bet v 1-specific IgE and the levels of IgE strongly correlated with those against Bet v 1 ( $\mathrm{r}=$ $0.82, \mathrm{p}<0.01)$. A diagnostic specificity of $100 \%$ and a sensitivity of $72 \%$ were observed for the related peanut seed storage protein allergens, Ara h 1, Ara h 2, Ara h 3 and Ara $\mathrm{h} 6$ as no IgE was detected in sera of control individuals (table 3).
Ackerbauer et al. 
Fig. 1. Correlation of serum IgE levels specific for Ara h 1, Ara h 2, Ara h 3, Ara h 8 and Ara $h 9$ determined by ImmunoCAP and ImmunoCAP ISAC. The linear regression coefficient $\mathrm{R}^{2}$ is given for each correlation analysis. Dotted lines indicate the 0.35 $\mathrm{kU}_{\mathrm{A}} / \mathrm{l}$ cut-off for ImmunoCAP and 0.3 ISU-E for ImmunoCAP ISAC.

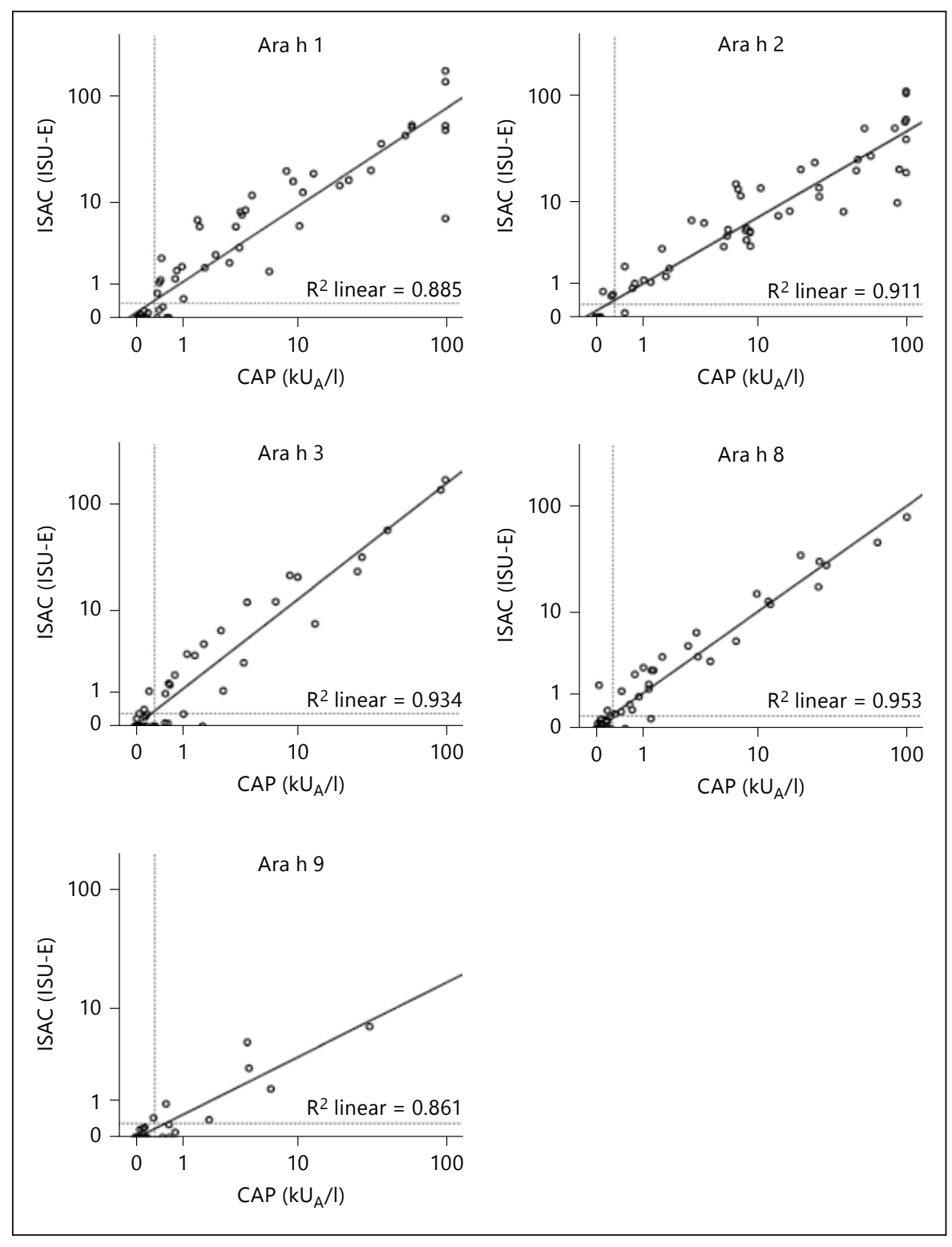

\section{Recognition Patterns of Specific IgE to Individual}

\section{Peanut Allergens}

The frequencies of sensitization and means of IgE levels for individual peanut allergens tested by ImmunoCAP and ImmunoCAP ISAC are shown in table 2. Due to high qualitative correlation between the two assays, the allergen sensitization patterns (fig. 2) and diagnostic sensitivities and specificities of individual peanut allergens are only shown for data obtained by ImmunoCAP ISAC (table 3 ).

Peanut-allergic patients were mainly sensitized to Ara h 2 and Ara h 6 (71\% for both), followed by Ara h 1 (62\%) and Ara h 8 (45\%). IgE reactivity to Ara h 3 was found in $35 \%$ and to Ara h 9 in $11 \%$ of peanut-allergic patients. In addition to Ara $\mathrm{h} 8$, the two other pollen allergy-associated allergens, Phl p 12 and CCDs, were recognized by 22 and $31 \%$, respectively. Both peanut-allergic patients with a positive history but negative peanut-specific $\operatorname{IgE}$ were sensitized to Ara h 8.

Polysensitization to more than 3 allergens was observed for $72 \%$ of patients and monosensitization to Ara h 8 was observed in 4 patients. Three patients were sensitized exclusively to Ara h 2 and Ara h 6. All sera containing Ara h 2-specific IgE were also positive for Ara h 6 and 


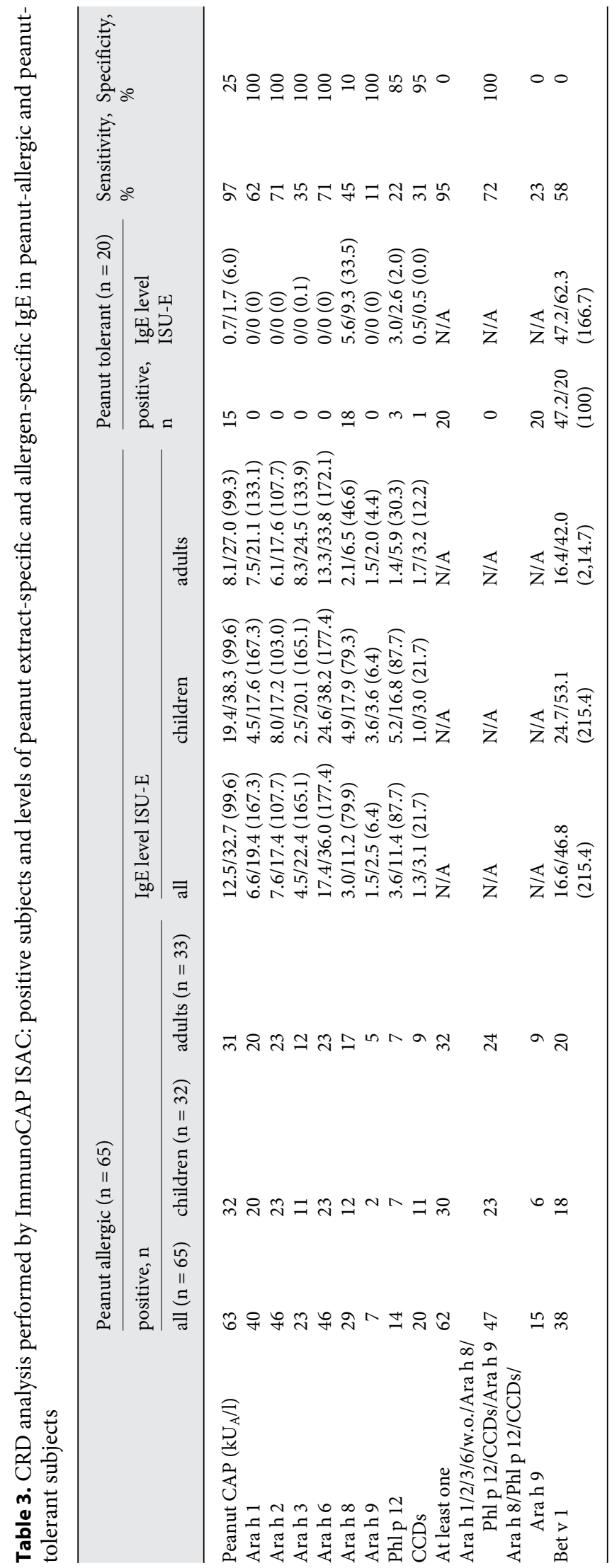

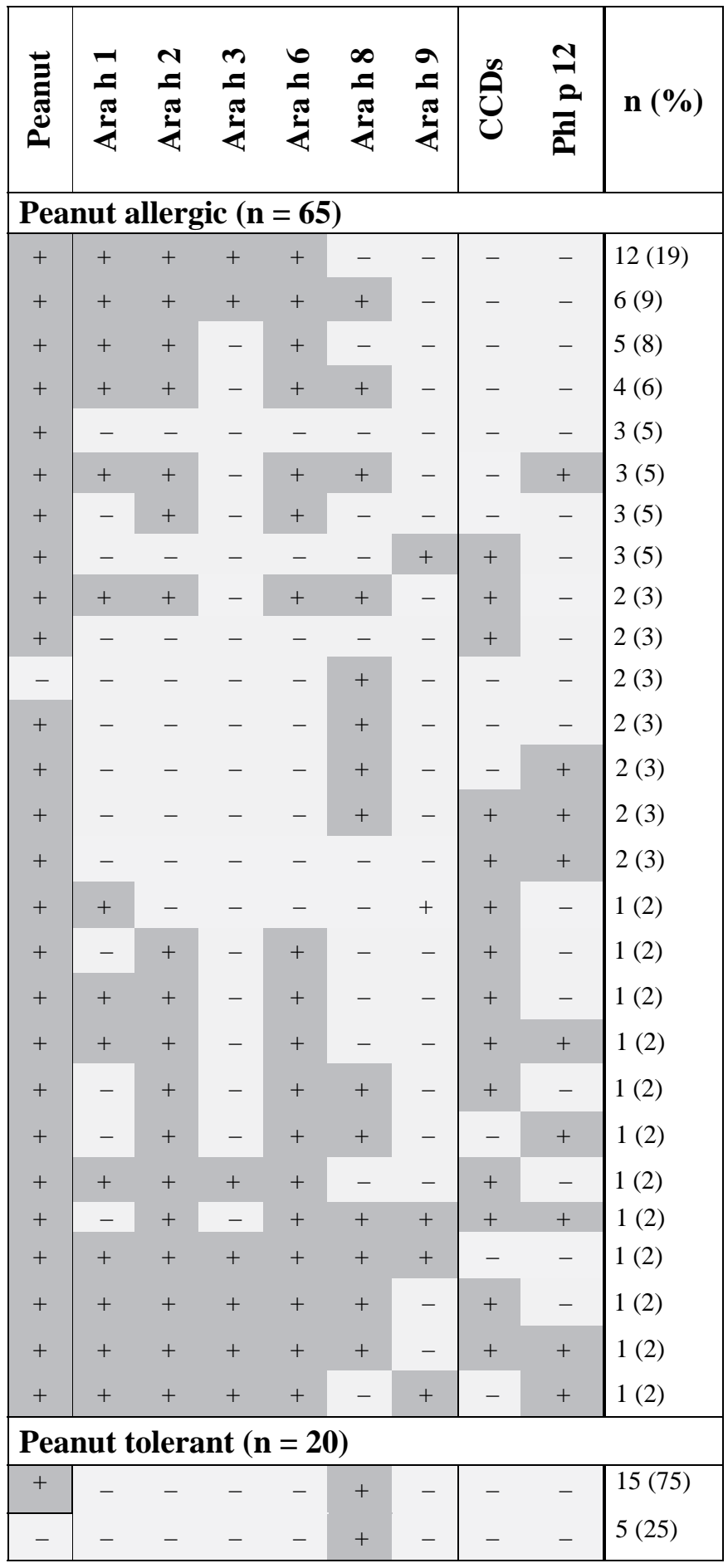

Fig. 2. IgE reactivity profiles to individual peanut allergens in 65 Austrian peanut-allergic patients and 20 peanut-tolerant but birch pollen-allergic patients.

\section{Footnote to Table 3}

IgE levels are presented as median/mean (range). N/A = Not applicable; w.o. = without. 
vice versa. Levels of Ara h 6-specific IgE (mean 36.0 ISU$\mathrm{E}$, range 177.4) were significantly $(\mathrm{p}<0.05)$ higher compared with IgE levels of Ara h 2-specific IgE (17.4 ISU-E, range 107.7; fig. 3). IgE levels to Ara h 9 were low with a specific IgE mean value of 2.5 ISU-E and associated with sensitization to other peanut components or to CCDs.

Forty-seven $(72 \%)$ peanut-allergic patients were positive for at least one of the peanut allergens belonging to families of seed storage proteins (Ara h 1, Ara h 2, Ara h 3 and Ara h 6). All but 1 of the patients sensitized to Ara h 1 also had IgE specific to Ara h 2, and all of the patients sensitized to Ara h 3 were also sensitized to Ara h 2 (fig. 2).

These patients also had a higher prevalence of anaphylaxis (i.e. cutaneous plus cardiorespiratory and/or GI involvement) after peanut exposure as compared with patients without sIgE to any of these allergens $(\mathrm{p}<$ 0.05 ). Ninety percent ( 28 of 31 ) of patients who experienced anaphylactic reactions after peanut exposure had specific IgE against Ara h 2. The 3 patients monosensitized to Ara h 2 and Ara h 6 had anaphylaxis, urticaria or OAS, respectively. Interestingly, 7 peanut-allergic patients were negative for all tested peanut allergens but positive for peanut extract ImmunoCAP. Two of those 7 were sensitized to CCDs and to CCDs and profilin, respectively.

When comparing symptom severity and sIgE levels for the different peanut allergens, we observed that patients experiencing anaphylaxis after exposure to peanut had significantly higher levels of $\operatorname{sIgE}(\mathrm{p}<0.01)$ against Ara h 1, Ara h 2, Ara h 3 and Ara h 6 compared with patients suffering from milder symptoms such as OAS (fig. 4). These patients also had significantly higher amounts of sIgE against Ara h 6 (sIgE mean 36.2 ISU$\mathrm{E}$, range 178.3) compared with patients showing reactions such as gastrointestinal symptoms, urticaria, dyspnea or angioedema (sIgE mean 19.3 ISU-E, range 102.2, $\mathrm{p}<0.05)$. The levels of Ara $\mathrm{h} 3$-specific IgE were significantly higher in the anaphylaxis (sIgE mean 15.4 ISU-E, range 165.1) and the systemic allergic symptom (sIgE mean 1.2 ISU-E, range 12.1) groups compared with the OAS group (sIgE mean 0.2 ISU-E, range 1.9, $\mathrm{p}<0.01)$.

All 4 patients monosensitized to Ara h 8 had OAS. Ara h 8 sIgE levels were the highest (sIgE mean 9.0 ISU-E, range 46.9) in patients suffering from OAS after exposure to peanuts, although this was not statistically significant (anaphylaxis group: sIgE mean 5.6 ISU-E, range 80.2, and systemic symptoms group: sIgE mean 3.2 ISU-E, range 31.2).

CRD in Austrian Peanut-Allergic Patients
IgE Sensitization Patterns to Homologues of Peanut Allergens

We evaluated IgE-binding patterns to homologues of peanut allergens which have been immobilized on the ImmunoCAP ISAC (fig. 5). Five of $40 \mathrm{rAra}$ h 1-positive sera contained IgE specific to both of two other members of the vicilin family, nGly $\mathrm{m} 5$ from soy and nJug $\mathrm{r} 2$ from walnut, while 9 recognized only nGly $\mathrm{m} 5$ and 8 only nJug $\mathrm{r} 2$. Six of the $8 \mathrm{rAra}$ h 1 - and nJug $\mathrm{r} 2$-positive sera showed a simultaneous positivity to the CCDs marker, nMUXF3. Sensitization to nJug $\mathrm{r} 1$ but not to rAra $\mathrm{h} 1$ was strongly associated with sensitization to nMUXF3 and also present in the group of atopic peanut-tolerant patients. Sensitization to other $2 \mathrm{~S}$ albumins was observed only with $20 \%$ of rAra h 2-and nAra h 6-positive sera, most of them sensitized to rJug $r 1$ from walnut $(8 / 46)$ followed by $n$ Ses i 1 from sesame (4/46) and rBer e 1 from Brazil nut (1/46). None of the rAra h 2- and nAra h 6-positive sera contained IgE specific to $\mathrm{nFag}$ e 2 from buckwheat. Leguminlike allergens such as nGly $m 6$ from soy were recognized by $65 \%$ of rAra h 3-positive sera. Only 2 Ara h 3-positive sera were positive to all three legumin-like allergens presented on the ImmunoCAP ISAC, namely nGly $\mathrm{m} 6$ from soy, rAna o 2 from cashew and nCor a 9 from hazelnut.

The three pollen Bet $\mathrm{v} 1$ homologues ( $\mathrm{rAln}$ g 1, rBet $\mathrm{v}$ 1 , rCor a 1.0101) presented on the ImmunoCAP ISAC were recognized by all rAra $h$ 8-positive sera, with one serum recognizing only Bet $\mathrm{v} 1$. The same sera also contained IgE specific to the Bet $\mathrm{v} 1$ homologues from foods such as rMal d 1 from apple, rCor a 1.0401 from hazelnut and rPru 1 from peach. Exceptions were rApi g 1 from celeriac and rGly $m 4$ from soy, which were recognized by 48 and $76 \%$ of the Ara h 8-positive sera, respectively. No differences in sensitization to Bet $\mathrm{v} 1$ homologues between peanut-allergic and peanut-asymptomatic atopic controls were observed (fig. 5). Among nsLTPs, all 7 rAra h 9-sensitized patients were also sensitized to rPru p 3, and 6 of them to nJug r 3. Only one serum contained IgE specific to pollen nsLTPs, such as nArt v 3 from Artemis$i a$ and nOle e 7 from olive pollen.

\section{Discussion}

In this study we analyzed the peanut allergen recognition patterns of 65 Austrian allergic patients with a convincing history of clinical immediate hypersensitivity to peanut. Specific IgE against peanut allergens was identified by the ImmunoCAP system for 5 single recombinant peanut allergens (Ara h 1-3, Ara h 8 and Ara h 9), and by

Int Arch Allergy Immunol 2015;166:13-24 DOI: $10.1159 / 000371422$ 
Fig. 3. Correlation (a) and comparison (b) of sIgE levels of Ara h 2 and Ara h 6 measured in ImmunoCAP ISAC.
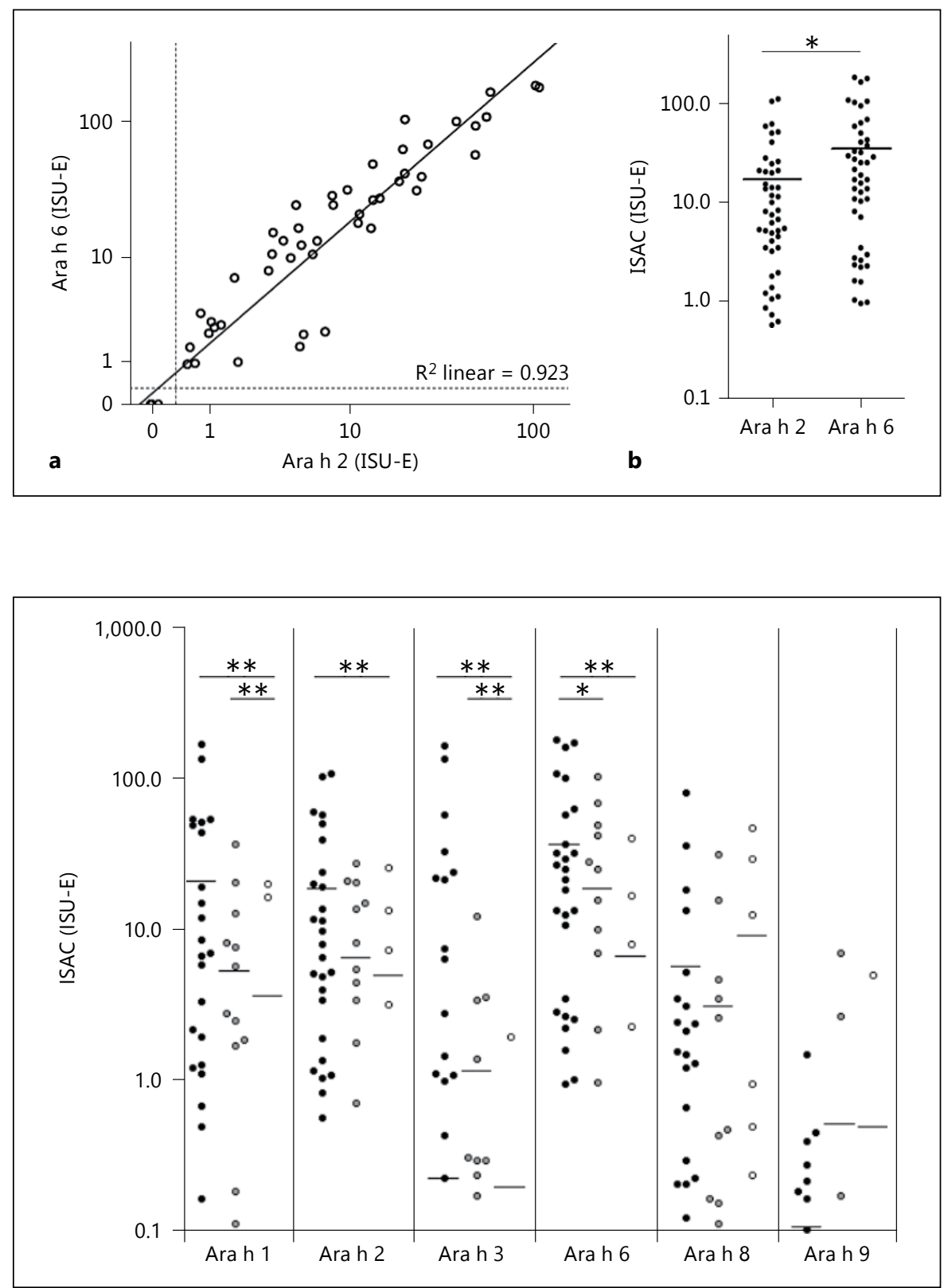

Fig. 4. Comparison of IgE levels specific for different peanut allergens measured by ISAC depending on symptom severity: anaphylaxis (black circles, $\mathrm{n}=31$ ), gastrointestinal symptoms only and other symptoms only (light grey circles, $\mathrm{n}=18$ ), and OAS (open circles, $\mathrm{n}=10$ ). ${ }^{*} \mathrm{p}<0.05,{ }^{* *} \mathrm{p}<$ 0.01 . the ImmunoCAP ISAC, which at present includes 6 peanut allergens (Ara h 1-3, Ara h 6, Ara h 8, Ara h 9). Furthermore, we compared the performance of those two assays to the traditional extract-based ImmunoCAP (f13) for in vitro diagnosis of peanut allergy.

Peanut allergen-specific IgE recognition patterns and diagnostic sensitivities of individual allergens determined by ImmunoCAP ISAC and singleplexed ImmunoCAP were highly comparable. Similarly, good correlations between these two techniques were obtained by Klemans et al. [2], who evaluated the sensitization patterns of 22 adults and 15 children with a positive double-blind, placebo-controlled peanut challenge. However, in our study we used convincing clinical history and sensitization to peanut to define peanut allergy, which could have influenced the diagnostic performance of the two assays.

The sensitivity of more than $90 \%$ of both CRD-based tests (at least one allergen positive) approached that of the peanut extract-based ImmunoCAP analysis (97\%). Our findings confirm observations from other peanut CRD studies that have demonstrated that this allergen panel enables the majority of peanut-allergic patients to be 
identified $[1,18]$. However, although the peanut extract was absolutely discriminative between patients with suspected peanut allergy and nonatopic controls, a positive sIgE result for peanut extract did not reflect the clinical relevance as it was also found in $75 \%$ of individuals without an apparent peanut allergy. The major cause for these false-positive peanut sIgE results seems to be the presence of cross-reactive IgE due to sensitization to Bet v 1, profilin or CCDs. In contrast, none of the sensitized but peanut-asymptomatic subjects demonstrated specific IgE reactivity to the peanut seed storage proteins Ara h 1, Ara h 2, Ara h 3 and Ara h 6, resulting in specificities of $100 \%$ for those allergens. In contrast, recent studies have shown that $26 \%$ of sensitized but tolerant patients' sera contained IgE specific to Ara h 2 [19], and that a 95\% probability of a positive peanut challenge could only be established for sIgE specific to Ara h 2 with a threshold of 42.2 $\mathrm{kU}_{\mathrm{A}} / \mathrm{l}$ and above [20]. We showed that $72 \%$ of Austrian peanut-allergic subjects had IgE specific to at least one of those four allergens. Most common was IgE specific to Ara h 2 and Ara h 6. All but 1 of the patients who were sensitized to Ara h 2 and Ara h 6 showed concomitant sensitization to Ara h 1 and/or Ara h 3. Our findings are in line with others showing that Ara h 2 is the most important peanut allergen $[1-6,11,21,22]$. Data about the prevalence and diagnostic value of Ara $h 6$ are rare, but in accordance with our results two studies showed that both peanut $2 S$ albumins are recognized by IgE from the same patients in almost all cases and that the diagnostic accuracy of IgE specific to Ara h 6 in adults is as good as for Ara h $2[1,7]$. The study performed by Klemans et al. [7] identified similar IgE levels for those two allergens when performing ImmunoCAP ISAC $[1,7]$. Interestingly, we found significantly higher levels of Ara h 6-specific IgE compared with IgE levels specific for Ara h 2, possibly due to the higher IgE affinity and IgE epitope availability of the purified natural Ara h 6 compared with recombinant Ara $h 2$ presented on the ImmunoCAP ISAC 112. In contrast to earlier studies, recent studies have increasingly suggested a positive correlation between the severity of symptoms and the level of specific IgE, especially to Ara h 2, but also to Ara h 1 and Ara h $3[3,6,9,23]$. We showed that patients experiencing anaphylaxis after exposure to peanut had significantly higher levels of sIgE against Ara h 1, Ara h 2, Ara h 3 and Ara h 6 as compared to patients suffering from milder symptoms such as OAS, although not all patients with severe reactions had high IgE levels to Ara $\mathrm{h} 2$. This is in line with the general statement to date that peanut-specific or Ara h 2-specific IgE levels do not clearly predict the severity of symptoms, and other

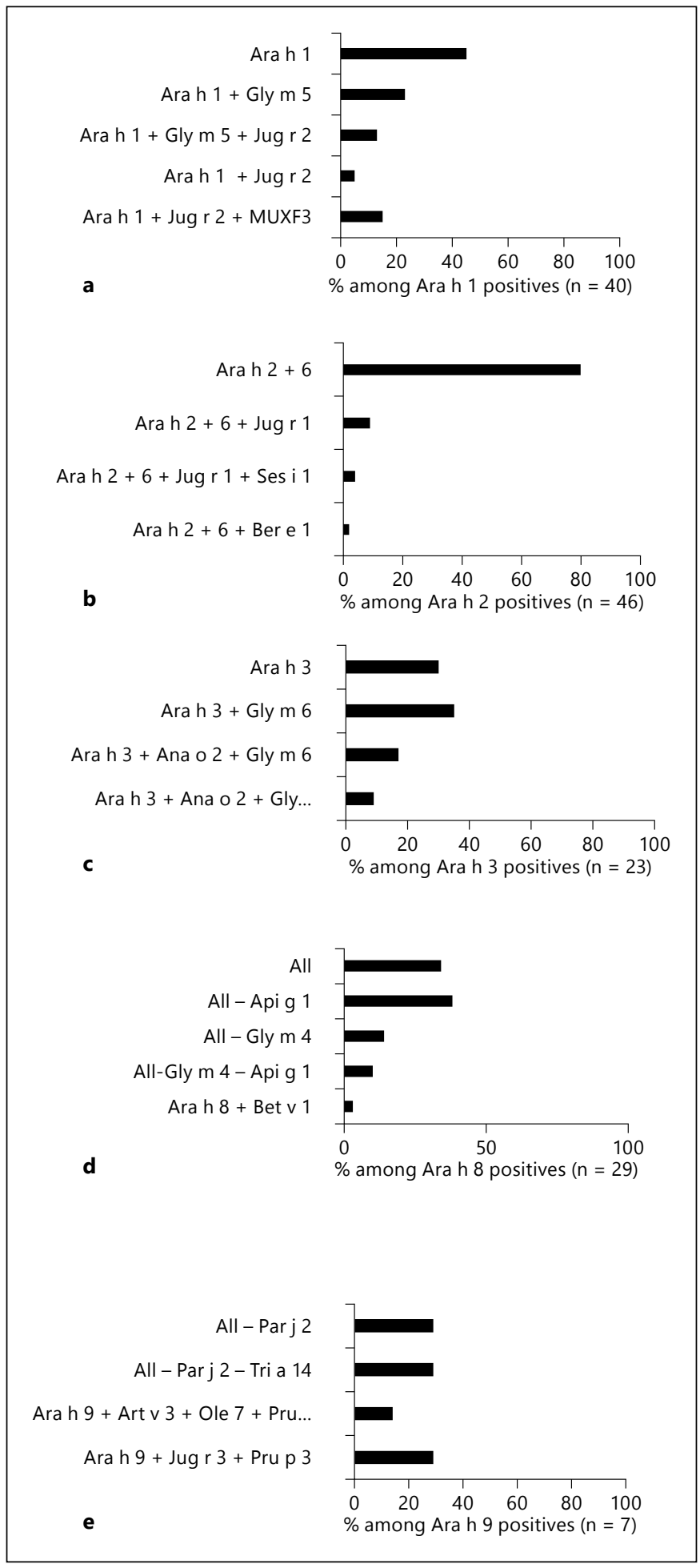

Fig. 5. Percentages of sensitization against homologues of peanut allergens in peanut-allergic patients. a Vicilins; MUXF3 is representative of CCDs. b $2 S$ albumins. c Legumins. d Bet v 1 homologues. All = Aln g 1, Act d 8, Api g 1, Ara h 8, Bet v 1, Cor a 1.0101, Cor a 1.0401, Gly m 4, Mal d 1 and Pru p 1. e nsLTPs. All = Ara h 9, Art v 1, Cor a 8, Jug r 3, Pla a 3, Ole e 7, Par j 2, Pru p 3 and Tri a 14. 
variables not related to the in vitro test might be more relevant, such as target organ reactivity, exposure to allergen dose or the state of health [16].

It emerged that patterns of IgE binding to peanut proteins may vary according to geographical location [1-6, $11,21,24]$. Sensitization to Ara h 8 is more prominent in peanut-allergic patients exposed to pollen from Fagales trees, such as birch or alder, and, according to recent studies from Sweden, isolated Ara h 8 sensitization is a marker of a mild reaction if any $[11,14]$. While $42-70 \%$ of peanut-allergic patients from Sweden, Japan or the Netherlands were sensitized to Ara h 8, only $19 \%$ of the patients from the USA and 2-7\% of Spanish patients had Ara h 8-specific IgE. In a study of children from a Swedish population-based cohort, only $18 \%$ of children with $\operatorname{IgE}$ reactivity exclusively to Ara h 8 had peanut allergy confined to mild symptoms [11]. In our study, $45 \%$ of peanut-allergic patients were sensitized to Ara $\mathrm{h} 8$, but only $12 \%(8 / 65)$, almost all with mild symptoms, had IgE reactivity to Ara $\mathrm{h} 8$ without concomitant sensitization to Ara h 1, Ara h 2, Ara h 3, Ara h 6 and Ara h 9. Four of them were sensitized exclusively to Ara h 8 and 4 also had IgE against profilin and/or CCDs. On the other hand, our results also clearly indicate that the presence of IgE antibodies to Ara $\mathrm{h} 8$ is in most cases not sufficient for the diagnosis of peanut allergy as most of the peanut-tolerant but birch pollen-allergic subjects showed IgE reactivity to Ara h 8 . Ara h 8 testing might add important additional diagnostic information, but evaluation of the medical history and, if applicable, oral food challenges are necessary for the accurate diagnosis of peanut allergy in these cases.

In our study, 7 subjects with allergic symptoms to peanut and with peanut extract-specific IgE displayed no IgE reactivity to any of the tested peanut allergens. The analysis of ImmunoCAP ISAC data revealed that 4 of them were sensitized to CCDs and 2 of those 4 were sensitized to profilins, but the remaining 3 subjects showed no sensitization to allergens with potential cross-reactivity to peanut. This observation, also reported by others $[11,14,18]$, indicates that additional peanut allergens might be implicated in peanut hypersensitivity in these patients. Although the peanut oleosins (Ara h 10, Ara h 11), peanut defensins (Ara h 12, Ara $\mathrm{h}$ 13) and an additional $2 S$ albumin (Ara h 7) have been identified as allergens, data about their clinical relevance for peanut-allergic patients are very scarce [25], suggesting that large-scale studies to evaluate the sensitization patterns to these components in peanut-allergic patients are required.

The evaluation of sensitization patterns to allergens related to peanut presented on the ImmunoCAP ISAC showed that about half of the patients' sera positive to Ara $\mathrm{h} 1$ and Ara $\mathrm{h} 3$ were also positive to related allergens from soy, Gly $\mathrm{m} 5$ and Gly $\mathrm{m} 6$. The high sequence identities between these allergens [26] indicate the presence of cross-reactive IgE antibodies. A study by Peeters et al. [27] revealed that $87 \%$ of peanut-allergic patients were sensitized to soy, but based on DBPCFC only $35 \%$ had symptoms to soy. IgE-binding to Jug $\mathrm{r} 2$ was in nearly all cases accompanied by IgE-binding to MUXF3, a marker of CCDs without clinical significance [28]. Interestingly, only $20 \%$ of Ara h 2- and Ara h 6-positive sera recognized other members of the $2 \mathrm{~S}$ family. Although sharing a wellconserved characteristic structure, $2 \mathrm{~S}$ albumins have low or almost no overall sequence identity, suggesting that IgE reactivity to these allergens indicates cosensitization rather than IgE cross-reactivity. On the other hand and in line with previous studies, high sequence identities among Bet $\mathrm{v}$ 1-homologous allergens were accompanied by a high IgE cross-reactivity, with similar frequencies of sensitization to Bet $\mathrm{v}$ 1-related allergens in peanut-allergic and peanut-tolerant patients $[29,30]$. Among nsLTP, sensitization to Ara h 9 was strongly associated with sensitization to Pru p 3, but not with the pollen nsLTPs, corroborating the hypothesis that Pru p 3 in peanut-allergic patients acts as a primary sensitizer [31].

For the in vitro diagnosis of peanut allergy especially in children, ImmunoCAP ISAC may provide additional benefit compared to the ImmunoCAP as it permits simultaneous assessment of specific IgE to six different peanut allergens, including the major allergen Ara $\mathrm{h}$ 6, with a minimal amount of serum $(0.04 \mathrm{ml}$ of serum for each individual allergen on ImmunoCAP vs. $0.02 \mathrm{ml}$ for ImmunoCAP ISAC). Recently, a high reproducibility of the ImmunoCAP ISAC analysis, in intra- as well as in inter-lab assays, has been shown for peanut allergens, suggesting the applicability of the technique for diagnosis and the follow-up of peanut-allergic patients [32]. However, due to its higher costs, the application of the ImmunoCAP ISAC is especially recommended in polysensitized patients for risk assessment or selection for immunotherapy, with the potential to not only identify symptom-related allergens, but also to rule out cross-reacting allergens without clinical relevance in one single measurement.

In conclusion, similar to other populations, the majority of Austrian peanut-allergic subjects are sensitized to Ara h 2. Additional tests for other peanut allergens, especially Ara h 8, should be considered when Ara h 2 is negative in peanut-symptomatic patients with birch pollen allergy. We found that results obtained with the ImmunoCAP ISAC assay are highly comparable to data obtained by CRD using the traditional ImmunoCAP. The
Ackerbauer et al. 
results of this study clearly demonstrate the greater ability of CRD in distinguishing between clinically relevant peanut allergy and asymptomatic sensitization to peanuts as compared with the diagnosis of peanut allergy based only on the peanut extract.

\section{Acknowledgments}

The authors would like to thank Phadia Austria, Thermo Fisher Scientific, for supporting this study, and Julia Lindenberger, $\mathrm{MSc}$, for performing ImmunoCAP ISAC analysis. Daniela Acker- bauer, Heimo Breiteneder (grant SFB 4608) and Karin HoffmannSommergruber (grant SFB 4603) were supported by the Austrian Science Fund.

\section{Disclosure Statement}

Thermo Fisher Scientific supplied the material for the ImmunoCAP and the ImmunoCAP ISAC required for the study. Heimo Breiteneder, Christine Hafner and Karin Hoffmann-Sommergruber received speaking fee lecture honoraria and Merima Bublin received a travel grant from Phadia Austria, Thermo Fisher Scientific.

\section{References}

1 Codreanu F, Collignon O, Roitel O, Thouvenot $B$, Sauvage $C$, Vilain AC, Cousin MO, Decoster A, Renaudin JM, Astier C, Monnez JM, Vallois P, Morisset M, Moneret-Vautrin DA, Brulliard M, Ogier V, Castelain MC, Kanny G, Bihain BE, Jacquenet S: A novel immunoassay using recombinant allergens simplifies peanut allergy diagnosis. Int Arch Allergy Immunol 2011;154:216-226.

2 Klemans RJ, Liu X, Knulst AC, Knol MJ, Gmelig-Meyling F, Borst E, Pasmans SG, Knol EF: IgE binding to peanut components by four different techniques: Ara $\mathrm{h} 2$ is the most relevant in peanut allergic children and adults. Clin Exp Allergy 2013;43:967-974.

3 Moverare R, Ahlstedt S, Bengtsson U, Borres MP, van Hage M, Poorafshar M, Sjolander S, Akerstrom J, van Odijk J: Evaluation of IgE antibodies to recombinant peanut allergens in patients with reported reactions to peanut. Int Arch Allergy Immunol 2011;156:282290.

4 Pedrosa M, Boyano-Martinez T, Garcia-Ara MC, Caballero T, Quirce S: Peanut seed storage proteins are responsible for clinical reactivity in Spanish peanut-allergic children. $\mathrm{Pe}$ diatr Allergy Immunol 2012;23:654-659.

5 Vereda A, van Hage M, Ahlstedt S, Ibanez MD, Cuesta-Herranz J, van Odijk J, Wickman M, Sampson HA: Peanut allergy: clinical and immunologic differences among patients from 3 different geographic regions. J Allergy Clin Immunol 2011;127:603-607.

6 Ebisawa M, Moverare R, Sato S, Maruyama N, Borres MP, Komata T: Measurement of Ara h 1-, 2-, and 3-specific IgE antibodies is useful in diagnosis of peanut allergy in Japanese children. Pediatr Allergy Immunol 2012;23:573581.

7 Klemans RJ, Knol EF, Bruijnzeel-Koomen CA, Knulst AC: The diagnostic accuracy of specific IgE to Ara $\mathrm{h} 6$ in adults is as good as Ara h 2. Allergy 2014;69:1112-1114.

8 Hong X, Caruso D, Kumar R, Liu R, Liu X, Wang G, Pongracic JA, Wang X: IgE, but not IgG4, antibodies to Ara $\mathrm{h} 2$ distinguish peanut allergy from asymptomatic peanut sensitization. Allergy 2012;67:1538-1546.

9 Klemans RJ, Otte D, Knol M, Knol EF, Meijer Y, Gmelig-Meyling FH, Bruijnzeel-Koomen CA, Knulst AC, Pasmans SG: The diagnostic value of specific IgE to Ara $\mathrm{h} 2$ to predict peanut allergy in children is comparable to a validated and updated diagnostic prediction model. J Allergy Clin Immunol 2013;131: 157-163.

10 Nicolaou N, Murray C, Belgrave D, Poorafshar M, Simpson A, Custovic A: Quantification of specific IgE to whole peanut extract and peanut components in prediction of peanut allergy. J Allergy Clin Immunol 2011;127: 684-685.

11 Asarnoj A, Moverare R, Ostblom E, Poorafshar M, Lilja G, Hedlin G, van Hage M, Ahlstedt S, Wickman M: IgE to peanut allergen components: relation to peanut symptoms and pollen sensitization in 8-year-olds. Allergy 2010;65:1189-1195.

12 Astier C, Morisset M, Roitel O, Codreanu F, Jacquenet S, Franck P, Ogier V, Petit N, Proust B, Moneret-Vautrin DA, Burks AW, Bihain B, Sampson HA, Kanny G: Predictive value of skin prick tests using recombinant allergens for diagnosis of peanut allergy. $\mathrm{J} \mathrm{Al-}$ lergy Clin Immunol 2006;118:250-256.

13 Flinterman AE, Knol EF, Lencer DA, Bardina L, den Hartog Jager CF, Lin J, Pasmans SG, Bruijnzeel-Koomen CA, Sampson HA, van Hoffen E, Shreffler WG: Peanut epitopes for IgE and IgG4 in peanut-sensitized children in relation to severity of peanut allergy. J Allergy Clin Immunol 2008;121:737-743.

14 Asarnoj A, Nilsson C, Lidholm J, Glaumann S, Ostblom E, Hedlin G, van Hage M, Lilja G, Wickman M: Peanut component Ara h 8 sensitization and tolerance to peanut. J Allergy Clin Immunol 2012;130:468-472.

15 Amoah AS, Obeng BB, Larbi IA, Versteeg SA, Aryeetey Y, Akkerdaas JH, Zuidmeer L, Lidholm J, Fernandez-Rivas M, Hartgers FC, Boakye DA, van Ree R, Yazdanbakhsh M: Peanut-specific IgE antibodies in asymptom- atic Ghanaian children possibly caused by carbohydrate determinant cross-reactivity. J Allergy Clin Immunol 2013;132:639-647.

16 Sicherer SH, Wood RA: Advances in diagnosing peanut allergy. J Allergy Clin Immunol Pract 2013;1:1-13.

17 Sicherer SH, Sampson HA: Food allergy: epidemiology, pathogenesis, diagnosis, and treatment. J Allergy Clin Immunol 2014;133: 291-307.

18 Dang TD, Tang M, Choo S, Licciardi PV, Koplin JJ, Martin PE, Tan T, Gurrin LC, Ponsonby AL, Tey D, Robinson M, Dharmage SC, Allen KJ: Increasing the accuracy of peanut allergy diagnosis by using Ara h 2. J Allergy Clin Immunol 2012;129:1056-1063.

19 Lopes de Oliveira LC, Aderhold M, Brill M, Schulz G, Rolinck-Werninghaus C, Clare Mills EN, Niggemann B, Naspitz CK, Wahn $\mathrm{U}$, Beyer K: The value of specific IgE to peanut and its component Ara $\mathrm{h} 2$ in the diagnosis of peanut allergy. J Allergy Clin Immunol Pract 2013;1:394-398.

20 Beyer K, Grabenhenrich L, Beder A, Kalb B, Ziegert M, Finger A, Harandi N, Schlags R, Gappa M, Puzzo L, Roblitz H, Millner-Uhlemann M, Busing S, Ott H, Lange L, Niggemann B: Predictive values of component-specific IgE for the outcome of peanut and hazelnut food challenges in children. Allergy 2015; 70:90-98.

21 Lin YT, Wu CT, Cheng JH, Huang JL, Yeh $\mathrm{KW}$ : Patterns of sensitization to peanut allergen components in Taiwanese preschool children. J Microbiol Immunol Infect 2012;45: 90-95.

22 Bublin M, Kostadinova M, Radauer C, Hafner C, Szepfalusi Z, Varga EM, Maleki SJ, Hoffmann-Sommergruber K, Breiteneder H: IgE cross-reactivity between the major peanut allergen Ara h 2 and the nonhomologous allergens Ara h 1 and Ara h 3. J Allergy Clin Immunol 2013;132:118-124.

23 Eller E, Bindslev-Jensen C: Clinical value of component-resolved diagnostics in peanutallergic patients. Allergy 2013;68:190-194. 
24 Suratannon N, Ngamphaiboon J, Wongpiyabovorn J, Puripokai P, Chatchatee P: Component-resolved diagnostics for the evaluation of peanut allergy in a low-prevalence area. Pediatr Allergy Immunol 2013;24:665-670.

25 Bublin M, Breiteneder H: Cross-reactivity of peanut allergens. Curr Allergy Asthma Rep 2014;14:426.

26 Chruszcz M, Maleki SJ, Majorek KA, Demas M, Bublin M, Solberg R, Hurlburt BK, Ruan S, Mattison CP, Breiteneder H, Minor W: Structural and immunologic characterization of Ara h 1, a major peanut allergen. J Biol Chem 2011;286:39318-39327.

27 Peeters KA, Koppelman SJ, Penninks AH, Lebens A, Bruijnzeel-Koomen CA, Hefle SL, Taylor SL, van Hoffen E, Knulst AC: Clinical relevance of sensitization to lupine in peanutsensitized adults. Allergy 2009;64:549-555.
28 Villalta D, Conte M, Asero R, Da Re M, Stella $\mathrm{S}$, Martelli P: Isolated IgE reactivity to native walnut vicilin-like protein (nJug $r$ 2) on IS$\mathrm{AC}^{\mathrm{TM}}$ microarray is due to cross-reactive carbohydrate epitopes. Clin Chem Lab Med 2013;51:1991-1995.

29 Ebo DG, Bridts CH, Verweij MM, De Knop KJ, Hagendorens MM, De Clerck LS, Stevens WJ: Sensitization profiles in birch pollen-allergic patients with and without oral allergy syndrome to apple: lessons from multiplexed component-resolved allergy diagnosis. Clin Exp Allergy 2010;40:339347.
30 Guhsl EE, Hofstetter G, Lengger N, Hemmer W, Ebner C, Froschl R, Bublin M, Lupinek C, Breiteneder $\mathrm{H}$, Radauer C: IgE, IgG4 and IgA specific for Bet v 1-related food allergens do not predict oral allergy syndrome. Allergy 2015;70:59-66.

31 Javaloyes G, Goikoetxea MJ, Garcia Nunez I, Aranda A, Sanz ML, Blanca M, Diaz Perales A, da Souza J, Esparza I, del Pozo V, Blazquez $A B$, Scheurer S, Vieths S, Ferrer M: Pru p 3 acts as a strong sensitizer for peanut allergy in Spain. J Allergy Clin Immunol 2012;130: 1432-1434.e3.

32 Martinez-Aranguren R, Lizaso MT, Goikoetxea MJ, Garcia BE, Cabrera-Freitag P, Trellez $O$, Sanz ML: Is the determination of specific IgE against components using ISAC 112 a reproducible technique? PLoS One 2014;9: e88394. 